\title{
The Gearbox Fault Diagnosis Based on Wavelet Transform
}

\author{
Jinyu Wang ${ }^{1, a}$, Dejian Kong ${ }^{2, b}$, Shi Dong ${ }^{3, c}$, Chao Wang ${ }^{4, d}$ \\ ${ }^{1,2}$ Department of Electrical \& Information Engineering, Northeast Petroleum University, Heilongjiang \\ Daqing, 163318, China \\ ${ }^{3}$ The Application Management Office of Information Center in the Second Oil Production \\ Plant,Heilongjiang Daqing, 163318, China \\ aemail: wangjydxl@126.com, bemail: kongdejian0154@163.com
}

Keywords: Wavelet Transform; Gearbox; Vibration; Fault-diagnosis

\begin{abstract}
The main gearbox is an important driving force for a pump. It needs to carry out regular monitoring. In this paper, it measures the vibration acceleration signal of a pumping unit's main gearbox, names them $1 \#$ and $2 \#$. After comparing the time course curve and spectral of the two main gearbox, it has found the 2\# gearbox's maximum amplitude is about 2.5 times than the $1 \#$. The narrowband signals are filtered by Hilbert transform to obtain the envelope of the narrow band signal. The envelope signals are significant different between the two gear boxes, $1 \#$ gearbox has more spectrum of frequency, and 2\# gearbox is more outstanding single frequency component. The results show that the presence of the 2\#gearbox has some minor faults. The 2\# gearbox is more wear than 1 \# gearbox, which explains the rationality of the vibration analysis.
\end{abstract}

\section{Introduction}

A pump's power plant is an equipment of four machines, which has biaxial propelled diesel-diesel combined propulsion. Two one each side of the diesel engine output single axial power through the main gearbox. The main gearbox is the main component of the power[1]. To vibration monitor it can detect faults, eliminate the hidden dangers and introduce the use of equipment, management and maintenance. However, the gearbox vibration signal is complicated. It has so much frequency components. So analysis and process the vibration signal is the key to fault diagnosis[2].

\section{Data Collection and Analysis}

After collecting the gearbox vibration signal, set the sample frequency to $12.8 \mathrm{kHz}$, collect continuous duration of $5.12 \mathrm{~s}$, collect 65536 points. The measuring points are arranged in $1 \#$ gearbox right free end of the pinion and 2\# gearbox right free pinion end, each measurement point has three directions of perpendicular, horizontal radial and horizontal axis[3]. The vibration acceleration time history is shown in Figure 1. For clarity, it only draws the front $0.2 \mathrm{~s}$ data. As can be seen, the more frequency components of acceleration, the more maximum acceleration reaches to $10 \mathrm{~m} / \mathrm{s}^{2}$. The different of the two gearbox vibration acceleration time history curve is less.

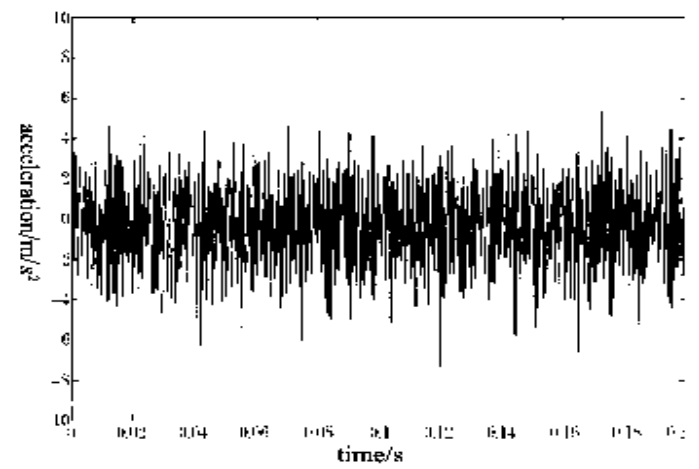

(a) $1 \#$ Gearbox result

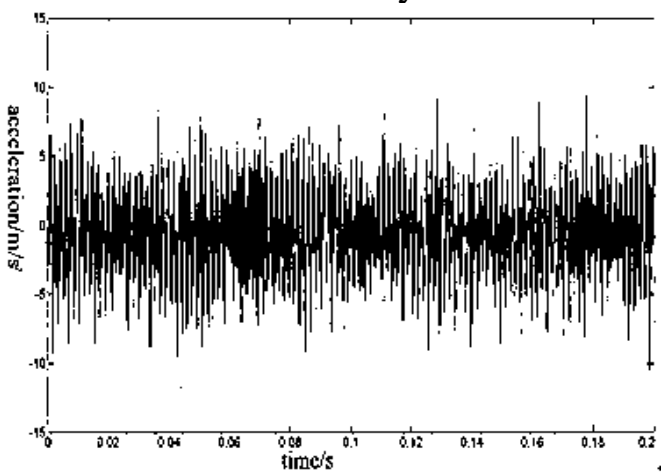

(b) 2\#Gearbox result

Fig.1. 1\# and 2\# Gearbox vertical acceleration time histories 
The vibration acceleration spectrum is shown in Figures 2. It can be seen from the spectrums, the acceleration spectrum consists of a wideband spectrum and a narrowband spectrum around the meshing frequency. It has found the 2\# gearbox's maximum amplitude is about 2.5 times than the $1 \#$, and the frequency which corresponds to the spectral value is close to the meshing frequency.

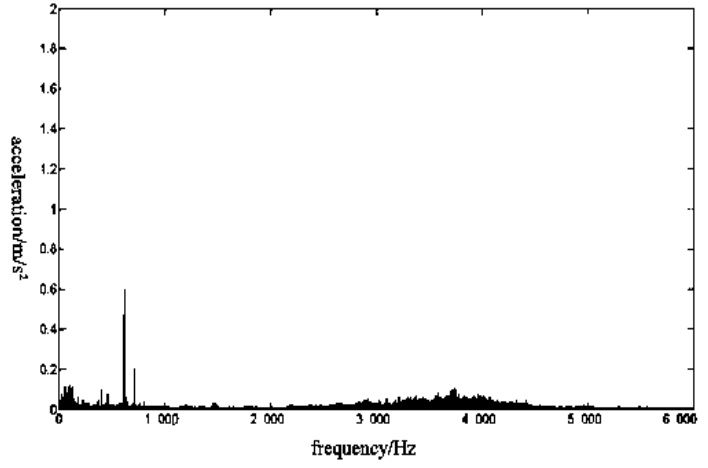

(a) 1 \# Gearbox result

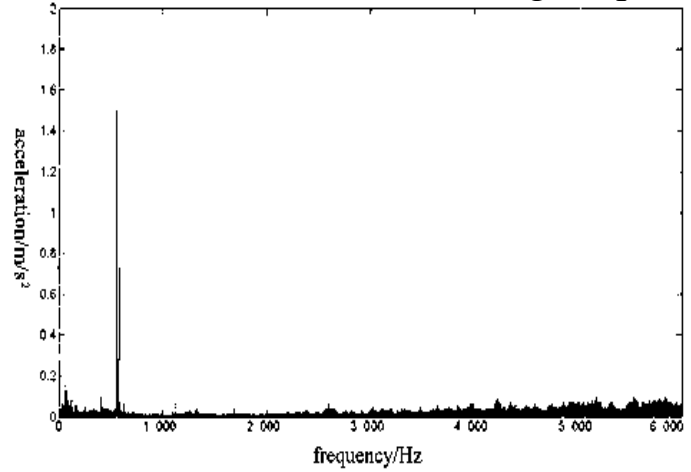

(b) 2\#Gearbox result

Fig.2. 1\# and 2\# Gearbox vertical acceleration time histories

\section{Analysis of Vibration Acceleration Signals Around the Gearbox Meshing Frequency}

\section{The Modulation Phenomenon at the Mesh Frequency}

During the processing of the gear transmission, the load, the stiff, the speed fluctuations and errors will change the gear vibration, resulting in the modulation phenomenon of meshing frequency[4]. The changing modulation signal can reflect in the change state of the gear, so that it can reflect the gearbox malfunction. Therefore, the meshing frequency modulation analysis is an important part of gearbox vibration condition monitoring and fault diagnosis.

\section{Band-pass filtering}

To move out the interferences from other frequency components, it is necessary for first band-pass filter, to remain the component frequencies in the vicinity of the mesh frequency. It designs a band-pass filter by MATLAB. The meshing frequency is $575 \mathrm{~Hz}$, the lower cut-off frequency of the filter is $512 \mathrm{~Hz}$, the upper cut-off frequency is taken to $640 \mathrm{~Hz}$ and the filter order is 1000. After using MATLAB fir1 filters, the results are shown in Figures 3. The filter causes the front 0.04 s delay distortion, so the portion of the signal may not be considered. As it can be seen, an amplitude modulation frequency phenomenon shows significant in the engagement. However, 1\# and 2\# gearbox meshing frequency modulated signal is significantly different. 2\# gearbox modulation signal peak is larger than 1\#; 2\# gearbox modulation signal has a significant prominent frequency component.

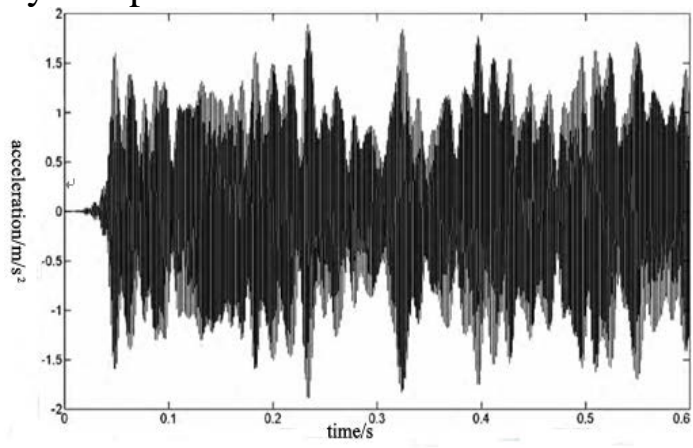

(a) 1 \# Gearbox result

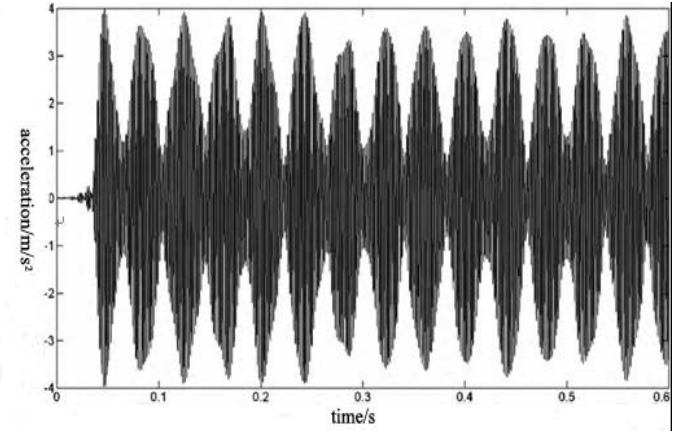

(b) 2\#Gearbox result

Fig.3. 1\# and 2\# Gearbox vertical acceleration time histories

\section{Converting the modulated signal based on the Hilebert analysis}

Using the Hilebert transform, turn the signal $x(t)$ into:

$\hat{x}(t)=\frac{1}{\pi} \int_{-\infty}^{+\infty} \frac{x(\tau)}{t-\tau} d \tau$

Order the plural: $z(t)=x(t)+j \hat{x}(t)$, then $z(t)$ is called the analytic signal of $x(t)$. The amplitude 
of $z(t)$ is corresponding to the envelope of $x(t)$. The Hilbert transformation is a basic tool for the analysis of the modulated signal. In MATLAB, the Hilbert transformation is $z=\operatorname{hilbert}(x)$. It is needed to be noted that the output $z$ is the analytic signal of the array $x$.The order of seeking $x$ envelope is $x_{e}=a b s(z)$.

The most substantial value of The engagement envelope of the right free end pinion of the 2\# gearbox vertical acceleration signal is $4 \mathrm{~m} / \mathrm{s}^{2}$, and $1 \#$ gearbox is $1.8 \mathrm{~m} / \mathrm{s}^{2}$. The envelope signal spectrum removing the direct current is shown in Figure 4.

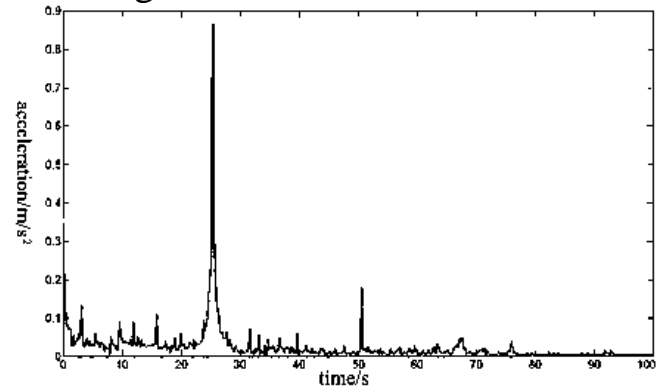

(a) 1 \# Gearbox result

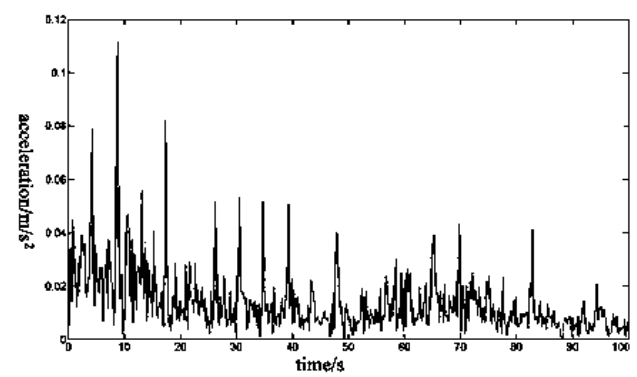

(b) 2\#Gearbox result

Fig.4. 1\# and 2\# Gearbox vertical vibration acceleration envelope signal spectrum near mesh frequency

The envelope signal spectrum frequency of $1 \#$ gearbox right pinion output is $8.8 \mathrm{~Hz}$. And the envelope signal spectrum frequency of 2 \# gearbox small right gear output is $25.4 \mathrm{~Hz}$, so the frequency component is very prominent. Comprehensive analysis of the results: the 2\# gearbox meshing vibration frequency is greater than 1\# gearbox. So there is a minor faulty of 2\# gearbox, the operation state is slightly worse than $1 \#$ gearbox. And the oil monitoring results also reflect that $2 \#$ gearbox wear slightly larger than $1 \#$ gearbox, as shown in Table 1 . The source of the prominent single frequency component requires further analysis. 2\# gearbox vibration intensity of $1.2 \mathrm{~mm} / \mathrm{s}, 1 \#$ gearbox is $1.3 \mathrm{~mm} / \mathrm{s}$. Two gearbox vibration intensity different is small, indicating that the fault is not serious, but also shows: Compared to the vibration intensity, the modulated signal frequencies are more sensitive to gearbox failure, is a better monitoring and diagnostic parameters

Table1 gearbox oil monitoring results

\begin{tabular}{c|c|c|c|c|c}
\hline device & $\mathrm{F}_{\mathrm{e}}$ & $\mathrm{C}_{\mathrm{r}}$ & $\mathrm{P}_{\mathrm{b}}$ & $\mathrm{C}_{\mathrm{u}}$ & $\mathrm{N}_{\mathrm{a}}$ \\
\hline 1\# gearbox & 11.3 & 0.2 & 9.5 & 11.7 & 86.9 \\
\hline 2\# gearbox & 21.7 & 0.6 & 18.2 & 20.5 & 176 \\
\hline
\end{tabular}

\section{Conclusion}

(1) The different between the two gearbox vibration acceleration signal is not obvious in time course curve, but the maximum amplitude differ of the spectrum is significant, that indicating a fault with the mesh frequency are closely related.

(2) In the vicinity of the meshing frequency band-pass filter, and then take the Hilbert transform envelope, two gearbox vibration signal difference is more obvious. The presence of a single frequency component was prominent in the $2 \#$ gearbox envelope signal, that results of failure, fluid analyzed for the presence of the gearbox also verified this conclusion.

(3) The vibration intensity different of the two gearbox is less, indicating a slight fault, but also shows the modulation signal at the frequency of engagement gearbox failure is more sensitive, is a better monitoring and diagnostic parameters.

(4) The $25.4 \mathrm{~Hz}$ is neither the $2 \#$ gearbox modulation frequency nor the diesel transfer frequency. The source of the frequency components still need more test data for further analysis to determine the specific source of the fault.

\section{References}

[1] Ding Kang, Li Weihua, Zhu Xiaoyong. Gears and Gearbox Fault Diagnosis Practical Technology. [M]. Beijing: Machinery Industry Press, 2005. 
[2] Fei-Sike Technology R\&D Center. MATLAB7 Auxiliary Signal Processing Technology and Applications. [M]. Beijing: Electronic Industry Press. 2005.

[3] Liu Shulin, Wang Jingdong, Li Fengming, etc. Shock and Vibration Handbook. [M]. Beijing: China Petrochemical Press. 2008.

[4] Wesley G. Zanardell,i Elias G. Strangas. Identification of Intermittent Electrical and Mechanical Fault in Permanent Ma-gentAC Drives Based on Time-frequency Analysis[J]. Industry Application IEEE Transaction on, 2007,43(3):971-980

[5] Jian-Da Wu, Jian-Ji Chan, "Faulted gear identification of a rotating machinery based on wavelet transform and artificial neural network", Expert Systems with Applications 36 (2009) 8862-8875. 